\title{
Vortex Structures in a Rotating BEC Dark Matter Component
}

\author{
N. T. Zinner \\ Department of Physics and Astronomy, Aarhus University, 8000 Aarhus C, Denmark \\ Correspondence should be addressed to N. T. Zinner, zinner@phys.au.dk \\ Received 28 April 2011; Accepted 4 August 2011 \\ Academic Editor: Robert Hallock \\ Copyright ( 2011 N. T. Zinner. This is an open access article distributed under the Creative Commons Attribution License, which \\ permits unrestricted use, distribution, and reproduction in any medium, provided the original work is properly cited.

\begin{abstract}
We study the effects of a dark matter component that consists of bosonic particles with ultralight masses in the condensed state. We compare previous studies for both noninteracting condensates and with repulsive two-body terms and show consistency between the proposals. Furthermore, we explore the effects of rotation on a superfluid dark matter condensate, assuming that a vortex lattice is formed as seen in ultracold atomic gas experiments. The influence of such a lattice in virialization of gravitationally bound structures and on galactic rotation velocity curves is explored. With fine-tuning of the bosonic particle mass and the twobody repulsive interaction strength, we find that one can have substructure on rotation curves that resembles some observations in spiral galaxies. This occurs when the dark matter halo has an array of hollow cylinders. This can cause oscillatory behavior in the galactic rotation curves in similar fashion to the well-known effect of the spiral arms. We also consider how future experiments and numerical simulations with ultracold atomic gases could tell us more about such exotic dark matter proposals.
\end{abstract}

\section{Introduction}

In recent decades we have witnessed a breakthrough in precision cosmology with observational data from measurements of distant Supernovae [1,2] and from WMAP [3] indicating that our universe is flat and dominated by nonstandard forms of matter and energy. The data tell us that only about $4 \%$ of the energy resides in the baryonic or luminous matter that we can observe through our telescopes. The rest of the energy is divided between the mysteries dark matter (26\%) and dark energy (70\%). The existence of dark matter (DM) was inferred earlier by its gravitational influence on the luminous matter in galaxies. Here the rotation velocity curves were found to disagree with expectations from general relativity. This lead people to introduce a dark matter halo around the galaxy that could explain the orbits. Models based on modification of the gravitational force are also being investigated but thus far such an explanation seems improbable [4].

The nature of this nonbaryonic DM is still a source of heavy theoretical and experimental investigation [5]. Over the years numerous matter particles have been suggested as candidates for the invisible dark matter. The most popular ones are the weakly interaction massive particles (WIMPs) which have very low reaction cross-sections with normal baryonic matter [6], although, since it is nonzero, there are many proposals and ongoing efforts to measure them directly [7]. One idea is that DM consists of scalar particles with extremely small masses, such as those appearing naturally as dilaton fields [8] in cosmological applications of string/Mtheory [9]. Ultralight DM was introduced in connection with a late-time cosmological phase transition [10] and assumed to be a Bose liquid in the condensate state [11]. Later it was shown that problems with small-scale structure in the otherwise successful cold dark matter (CDM) model could be remedied by a Bose-Einstein condensate (BEC) DM component [12-15], since it will behave as nonrelativistic $\mathrm{CDM}$ with a large quantum coherence length, avoiding the cusp behavior of DM halos found in simulations [12]. In $[11]$, and more recently in $[16,17]$, galactic rotation curves were calculated for BEC DM and compared to observations of dwarf and spiral galaxies.

Here we address the effects of rotation under the assumption that the dark matter halos are Bose-Einstein condensates (BECs) of ultralight particles. When a BEC is rotated at a rate exceeding some critical frequency, quantized vortices can be formed [18]. The effects of vortex formation in galaxy halos have been addressed in $[19,20]$. In [19], 
the vortex density of the Andromeda Galaxy M31 was considered, whereas [20] discusses a vortex as the cause of the flattened galaxy rotation curve. There has also been some interest in analogues between rotating spacetimes and superfluid systems [21]. The latter is a continuation of recent claims by Chapline and collaborators that black hole event horizons represent quantum phase transitions as seen in Bose fluids and therefore should not be regarded as a breakdown of classical general relativity [22].

Vortex lattices are seen in experiments with BEC [2326] when the sample is rotated at rates above the critical frequency. In those systems one has a superfluid singlecomponent BEC of alkali atoms at very low temperature with a two-body repulsive interaction described by the scattering length. Recently it was shown how an array of lasers can actually induce gravity-like interactions by averaging over the usual electromagnetic dipole-dipole force [27]. This would in principle allow one to study the gravitational dynamics of BEC matter in the laboratory. Rotating such a system would allow us to study the properties of vortices when long-range $1 / r$ attraction is present and perhaps reveal how structures are formed. An alternative and likely more feasible way to approach the issue is through numerical simulations. Extensive theoretical and experimental work in ultracold atomic gases has shown that Bose-Einstein condensates are accurately described by the nonlinear Gross-Pitaevskii equation [18]. Numerical simulations of systems governed by this equations including rotation and a gravitational potential would therefore presumably yield information on structure formation and vortex dynamics.

In this paper we want to explore some effects of rotating dark matter halos that consist of ultralight bosonic particles in the condensed state. This sort of dark matter would be superfluid and if rotated above the critical angular velocity it would allow the formation of a vortex lattices in the halo cloud. Section 2 considers the noninteracting case, whereas bosons with repulsive self-interactions are discussed in Section 3. Effects of rotation on superfluid condensates are considered in Section 4 in the light of previous works addressing this possibility in connection with halos of spiral galaxies. In Section 5 we explore the influence of vortex lattices in dark matter halos for early structure formation, in the virialization of gravitationally bound structures, and on the galactic rotation velocity curves. We compare the rotation curves in our ultralight BEC dark matter model to some observations of spiral galaxies and find that wiggles in the rotation curves can be accommodated in our model within certain parameter regimes. An ultralight BEC dark matter component can thus be an additional source of oscillatory behavior in the rotation curves, although the main source is presumably the baryonic matter in the spiral arms to which it is correlated $[28,29]$. Section 6 contains a discussion of some issues related to the fine-tuning of the mass of the bosonic particles and of the interaction strength of the repulsive two-body term. Some comparison between the presented astrophysical ideas and the current status of experiments and numerical simulations with ultracold atomic gases are given in Section 7. Possible ways to test such exotic dark matter proposals in the laboratory or on a computer are suggested. Finally, Section 8 contains a short summary and conclusions.

\section{Ultralight BEC Dark Matter}

In this section we consider the possibility of a BEC of ultralight particles as the sole dark matter component. This was first suggested in [11] and further explored in [12, 19]. The idea is that ultralight particle $m \ll 1 \mathrm{eV}$ will have very large de Broglie wavelengths which means that quantum statistical effects are important and macroscopic coherent lumps of matter can emerge. As shown in [19], these light Bose particles will have a transition temperature to the condensed state that is of order $T_{c} \sim 2 \cdot 10^{9} \mathrm{~K}$, which is the expected temperature in the Universe after about 1 second. (This estimate of the critical temperature is based on comparing the thermal de Broglie wavelength [18] to the mean interparticle distance. For condensation to set in these two quantities must be similar, giving the relation $k_{B} T_{c} \sim h c(\rho / m)^{1 / 3}$ where $\rho$ is the mean density of the Bosonic matter [19]. A more detailed calculation of the transition temperature for a uniform Bose gas yields the expression $k_{B} T_{c} \sim \hbar^{2}(\rho / m)^{2 / 3} / m$. Using the latter relation yields an even higher value for $T_{c}$ and does not alter the argument in the text that the Bose particles are expected to be in the condensed state at the present epoch.) The fall of the temperature with the expansion means that an overwhelming majority of the Bose particles will be found in the condensate state. For the moment we will assume that the particles are noninteracting (we will relax this assumption below) and therefore only gravity acts on the system. Following [12], we now resort to Jeans instability analysis to estimate our parameters. The growing mode under gravity is $e^{\gamma t}$ with $\gamma^{2}=4 \pi G \rho$, whereas the free field will be oscillatory: $e^{-i E t}$ with $E=k^{2} / 2 m$. The latter can be written as $e^{\gamma t}$ with $\gamma^{2}=-\left(k^{2} / 2 m\right)^{2}$. Noting that this is like normal Jeans analysis with sound speed $c_{s}^{2}=k / 2 m$ we have $\gamma^{2}=4 \pi G \rho-\left(k^{2} / 2 m\right)^{2}$. Setting this to zero, we get the Jeans scale

$$
\begin{aligned}
r_{J} & =\frac{2 \pi}{k_{J}}=\pi^{3 / 4}(G \rho)^{1 / 4} m^{-1 / 2} \\
& =55 m_{22}^{-1 / 2}\left(\frac{\rho}{\rho_{b}}\right)^{-1 / 4}\left(\Omega_{m} h^{2}\right)^{-1 / 4} \mathrm{kpc},
\end{aligned}
$$

where $m_{22}=m / 10^{-22} \mathrm{eV}$ and the background density is $\rho_{b}=2.8 \cdot 10^{11} \Omega_{m} h^{2} M_{\odot} \mathrm{Mpc}^{-3}$. Below the Jeans scale the perturbations will be stable and above it they will behave as ordinary CDM [12]. The stability below the Jeans scale is guaranteed by the uncertainty principle. If the particles are confined further, their momenta will increase and oppose the gravitational contraction.

From the Jeans scale in (1) we can see that the mass has to be extremely small for the current scenario to be responsible for galaxy-size structures at present. A typical galaxy has a matter density of about $200 \rho_{b}$ and a radius of order $r \sim 10 \mathrm{kpc}$. Using $\Omega=0.3$ and $h=0.7$ in (1), we see that a mass of precisely order $m \sim 10^{-22} \mathrm{eV}$ is needed for the condensed particles. As noted in [12], the observational evidence can accommodate even lighter fields $m \lesssim 10^{-33} \mathrm{eV}$ 
in quintessence models. Reference [12] proceeds with onedimensional simulations and shows that such ultralight Bose particles could actually help solve cusp problem in dark matter halos by suppressing small-scale linear. This was confirmed in more detail in [30].

As mentioned in the introduction, the rotation curve of galaxies is an important feature that dark matter explains very well. In [19] the ultralight BEC dark matter scenario was used to predict the rotation curve and the results were compared to observations from the Andromeda galaxy M31. The model was found to agree with the data if the boson mass was in the range $m \sim 10^{-24}-10^{-23} \mathrm{eV}$, in rough agreement with the analysis in [12].

\section{Including Self-Interaction}

There has been a number of investigations into dark matter models with Bose particles that consider the (likely) more realistic case of bosons with repulsive self-interactions [13, 14]. The motivation for this was the problem of too much subgalactic structure that was mentioned above. The typical case considered is that of a quartic self-interaction $\lambda \phi^{4}(\lambda>$ 0 ) which is the generic textbook example of an interacting field theory. From an atomic physics point of view the quartic case is also very interesting as the mean field approximation yields the Gross-Pitaevskii equation (GPE) for the condensate state that has proven very useful in describing BEC experiments [18].

Even more interesting is the fact that there is actually an exact solution for the case of a self-gravitating Bose gas with a repulsive quartic term $[13,17]$. This comes about since in this case the GPE can be recast into the Lane-Emden equation with polytropic index $n=1$ which has the analytic solution

$$
\rho(r)=\rho_{0} \frac{\sin \left(r / r_{0}\right)}{r / r_{0}}, \quad r_{0}=\sqrt{\frac{K}{2 \pi G}} .
$$

$K$ comes from the equation of state: $p=K \rho^{2}$. This latter relation can be found by simply estimating the ground-state energy of a Bose condensate with repulsive self-interaction [18]. In atomic physics, one would typically relate the interaction strength (specified by $\lambda$ above) to the scattering length for two-body scattering at very low energy. In our current notation this yields $K=g n / 2 m^{2}$, $n$ being the particle density and $g=4 \pi \hbar^{2} a / m$ is called the interaction coupling constant [18]. The sound velocity in the gas is given by $c_{s}^{2}=g n / m$. The observed dark matter halos do not have the profile given by (2) but, as noted in [13], there could be nonzero momentum particles present that would allow a more realistic power-law behavior outside the core.

In this repulsive self-interaction scenario one can estimate the mass of the Bose particles by using the first zero of (2), $R=\pi r_{0}$, which is assumed to be the halo radius. Combining all the formula given above yields

$$
m=\left(\frac{\pi^{2} \hbar^{2} a}{G R^{2}}\right)^{1 / 3}=6.73 \cdot 10^{-2} a^{1 / 3} R^{-2 / 3} \mathrm{eV},
$$

where $a$ is measured in Fermi and $R$ in kpc. Taking $R \sim 10 \mathrm{kpc}$ and $a$ between $1 \mathrm{fm}$ and $1 \mathrm{~nm}$ (typical atomic magnitudes [18]), we obtain masses in the range from $m \sim$ $10 \mathrm{meV}$ to $1 \mathrm{eV}$. We immediately notice that there are many orders of magnitude away from the noninteracting case of the last section. A mass of $m \sim 10^{-22} \mathrm{eV}$ would require an extremely low scattering length of $a \sim 3.3 \cdot 10^{-61} \mathrm{fm}$ (keeping the same halo size). This is of course extremely small and borders on the noninteracting case which tells us that there is consistency with the results presented in the previous section.

At this point one might wonder if the critical temperature estimates given earlier will be altered significantly by the fact that the bosons now have a repulsive two-body interaction. In the context of the uniform Bose gas model, this has been addressed in [31] where is was shown that the change in the critical temperature obeys $\delta T_{c} / T_{c} \sim a n^{1 / 3}$. If we measure $a$ in Fermi, $m$ in $\mathrm{eV}$, and $\rho$ in $10^{-23} \mathrm{~g} / \mathrm{cm}^{-3}$ (typical halo density), then we get $\delta T_{c} / T_{c} \sim 2 \cdot 10^{-10} a(\rho / m)^{1 / 3}$. We therefore see that the correction to $T_{c}$ is extremely small for both $m \sim 10^{-2}-$ $1 \mathrm{eV}$ and for ultralight $m \sim 10^{-22} \mathrm{eV}$ (remembering that $a \propto$ $m^{3}$ according to (3)). We therefore see that the estimate $T_{c}>$ $2 \cdot 10^{9} \mathrm{~K}$ given earlier will not be altered by the repulsive selfinteraction.

The considerations in this and the previous section rely on Newtonian gravity, and general relativistic effects could modify the results. A general relativistic description of the BEC dark matter scenario is discussed in [17], particularly the effects on the rotational curves of galaxies. It was found that rotational curves of a number of galaxies could be well reproduced, although the Newtonian analysis already produces a tangential velocity of test particles at the halo radius of $365 \mathrm{~km} / \mathrm{s}$, consistent with observations. Note also that we are tacitly assuming that the ultralight Bose particles are nonrelativistic which was shown to be a very good approximation in $[11,19]$. We therefore restrict our discussion to Newtonian gravity here.

\section{The Effects of Rotation}

Several studies have addressed the effects of rotation on BEC dark matter. In [17] there is a brief discussion of the effect on the Lane-Emden equation, whereas [20] consider a BEC of axions with a single vortex arising from global rotation in the early universe. This latter scenario is, however, less likely to occur since the global rotation rate of the universe can be estimated from various observations and is likely very small if nonzero [32]. The formation of vortices in condensates is known from atomic physics [18]. For superfluid Bose systems any angular momentum imparted will reside in quantized vortices that constitute small regions where the superfluid density goes to zero on a scale comparable to the coherence length (to be discussed below). As already noted in [13] BEC dark matter with self-interactions will actually constitute a superfluid.

A superfluid will only form these vortices when the rotation rate exceeds a critical frequency $\Omega_{c}$. For a sample of size $R$ with coherence length $\xi$ the critical value is [18]

$$
\Omega_{c}=\frac{\hbar}{m R^{2}} \ln \left(\frac{1.46 R}{\xi}\right)=\frac{6.21 \cdot 10^{-17}}{m_{22} R^{2}} \ln \left(\frac{1.46 R}{\xi}\right),
$$


where $m_{22}=m / 10^{-22} \mathrm{eV}$ and $R$ is measured in kpc. This critical frequency is based on the Gross-Pitaevskii equation and the coherence length $\xi$ is given by the self-interaction through $\xi=1 / \sqrt{8 \pi a n}$ with $n$ the particle density and $a$ the scattering length. The formation of vortices has been experimentally demonstrated in superfluid ${ }^{4} \mathrm{He}$ [23]. Later they were produced in dilute BEC alkali atom gases [2426] where beautiful lattices were created with more than 100 vortices in regular alignment. These experiments also showed a density profile that had dips at the vortex positions, confirming the theoretical expectation of density drops in the vortex core mentioned above, although the actual experiments had cores with small but nonzero density. The expected increase in the number of vortices with increasing rotation frequency was also observed. Theoretically, the vortex line density is given by $n_{v}=m \Omega / \pi \hbar$ which yields a total number of vortices $N_{v}=\pi R^{2} n_{\nu}$.

The idea of similar vortex formation in rotated BEC dark matter was first considered in [19]. Here the rotation rate of the Andromeda galaxy M31 was calculated and compared to the critical frequency. M31 was found to rotate at a rate vastly above $\Omega_{c}$, and vortices were therefore suggested as a possible consequence. The total number of vortices for M31 was found to be around $N_{v} \sim 340$. As a possible means of detecting these vortices, [19] suggested gravitational lensing or polarization effects from frame dragging of light from distant background sources. The use of gravitational lensing to infer the possible existence of a BEC dark matter component was also suggested and explored in [17].

As already mentioned above, the halo radius can be taken as the first zero of the solution in (2), that is, $R^{2}=$ $\pi^{2} \hbar^{2} a / \mathrm{Gm}^{3}$. In the scalar field treatment with interaction $\lambda \phi^{4}$, the corresponding size estimate is $R^{2}=\pi^{2} \lambda \hbar^{3} / 8 \pi G m^{4} c$ $[15,33]$. Equating these, we obtain $\lambda=8 \pi a m c / \hbar=1.3$. $10^{-29}(\mathrm{a} / 1 \mathrm{fm})\left(\mathrm{m} / 10^{-22} \mathrm{eV}\right)$. Using $m=10^{-22} \mathrm{eV}$, a halo mass density $\rho_{\text {halo }} \sim 10^{-23} \mathrm{~g} / \mathrm{cm}^{-3}$, and assuming that $\xi \sim$ $1 \mathrm{kpc}$, we find $\lambda \sim 10^{-92}$, a very small value. As noted earlier, the repulsive self-interaction is not likely to influence the gravitational formation of the halo [15]. However, as discussed above such small values give large vortex cores when $\Omega \gg \Omega_{c}$. In [33], fits to rotation curves were presented and it was found that $m^{4} / \lambda \sim 50-75 \mathrm{eV}^{4}$. For $m=$ $10^{-22} \mathrm{eV}$ this gives $\lambda \sim 10^{-90}$, close to the present estimate. Importantly, our smaller $\lambda$ actually fulfills the Big Bang Nucleosynthesis (BBN) bounds discussed in [33].

\section{A Dark Matter Vortex Lattice}

If dark matter contains a component of condensed BEC particles that is superfluid and if the halos are rotating then it is not inconceivable that there can be vortex formation as discussed above. However, the quantized vortex discussion of [19] makes an important assumption about the coherence length, $\xi$, entering $\Omega_{c}$ in (4). $\xi$ is taken to be of kpc size. This is based on the noninteraction arguments of Section 2 . With no self-interaction there is only the gravitational scale $\hbar^{2} / G M m^{2}$ available, which becomes of galactic size for masses $m \sim 10^{-22} \mathrm{eV}$. However, when including selfinteractions as in Section 3 through a scattering length $a$, there is also a scale given by $\xi=1 / \sqrt{8 \pi a n}$, which is the usual Gross-Pitaevskii coherence length. The latter coincides with the characteristic length over which the density is expected to go to zero in a vortex.

In the following discussion we will make the assumption that it is actually the coherence length $\xi$ that determines the vortex core size. This is reasonable since the vortices are local entities and as such should depend on the local interactions. From this point of view the gravitational scale $\hbar^{2} / G M m^{2}$ merely serves to determine the total halo cloud size. The coherence length can be written as

$$
\xi=2.73 \cdot 10^{-32} m_{22}^{1 / 2} a^{-1 / 2} \rho_{\mathrm{DM}}^{-1 / 2} \mathrm{kpc},
$$

where $\rho_{\mathrm{DM}}$ is the dark matter halo mass density measured in $10^{-23} \mathrm{~g} / \mathrm{cm}^{3}, a$ is in Fermi, and $m_{22}=m / 10^{-22} \mathrm{eV}$. The measure for $\rho_{\mathrm{DM}}$ originates from assuming that a halo has a radius of about $10 \mathrm{kpc}$ and contains about $3 \cdot 10^{11} M_{\odot}$ of mass giving a density of about $1.74 \cdot 10^{-23} \mathrm{~g} / \mathrm{cm}^{3}$. The front factor in (5) seems exceedingly small and it would seem that any vortices on this scale would be completely irrelevant to the galaxy structure. However, we recall from the mass estimate in (3) that $a$ also needs to be extremely small. Inserting a value of $a \sim 10^{-61} \mathrm{fm}$ (corresponding to $m \sim 10^{-22} \mathrm{eV}$ ) actually gives $\xi \sim 0.05 \mathrm{kpc}$, so that the vortex size is subgalactic but sizable. This demonstrates that vortex structures could be important for the structure of rotating galaxies, and we will discuss possible effects below.

Before we consider vortex lattice effects on structure and evolution, we now briefly compare our suggestions with the observational data from M31. Studies in [19] estimate the critical frequency to be about $\Omega_{c}=2 \cdot 10^{-19} \mathrm{rad} / \mathrm{s}$ for M31 (using $R \sim 150 \mathrm{kpc}$ and $m \sim 2 \cdot 10^{-23} \mathrm{eV}$ ). The estimate for the actual rotation frequency of M31 based on observations is given as $\Omega=5 \cdot 10^{-17} \mathrm{rad} / \mathrm{s}$, so that the assumption $\Omega \gg \Omega_{c}$ is justified and a vortex lattice is therefore possible. However, in [19] the coherence length used in (4) was taken to be $\xi$ $30 \mathrm{kpc}$ (the gravitational length scale for the noninteracting condensate discussed above). We are assuming that the coherence length scale for vortex formation is that associated with the two-body repulsion. This is, however, not a serious obstruction since it enters only in the logarithmic term in (4). Using the value quoted above of $\xi \sim 0.05 \mathrm{kpc}$ in the critical frequency would therefore only amount to about a factor of five increase which would not jeopardize the relation $\Omega \gg$ $\Omega_{c}$. Furthermore, changing the two-body repulsion (through changes in $a$ ) would not be severe for the same reason.

5.1. Influence on Early Structure Formation. For the vortex lattice to appear in the current scenario with BEC dark matter we need rotation. One can assume that rotation is a primordial feature of the universe. This possibility has been explored by many authors [34-37]. However, this is at odds with considerations of $\mathrm{CMB}$ anisotropies [38], which suggest that the primordial rotation rate is very small [32]. The rotation that one finds in galaxies is therefore not considered to be primordial and is believed to arise from gravitational tidal torque forces during the growth of nonrotating initial perturbations toward virialized galactic structures. This way 
of generating angular momentum is well studied [39-41] and widely accepted as a key mechanism.

Although primordial rotation seems out of the question, we will briefly consider what would be the result if it was found that our universe could have been rotating in early epochs. More precisely we want to explore what would be the effect of a rotation rate large enough to support a vortex lattice at the time of decoupling when baryonic perturbations can start to grow. We can argue in the usual way that when baryonic perturbations can finally grow, they will do so under the influence of a potential well that is created by the dark matter perturbations. Assuming that the baryons themselves provide a negligible contribution to the background density, a Jeans analysis tells us that the baryonic density contrast in the linear regime obeys [42]

$$
\ddot{\delta}_{B}+\frac{4}{3 t} \dot{\delta}_{B}+\left(\frac{k_{B} T}{m_{p}}\right) \frac{k^{2}}{a^{3}} \delta_{B}=\frac{2}{3} \frac{\delta_{\mathrm{DM}}}{t^{2}},
$$

where $t$ is cosmic time, $a$ is the scale factor, $T$ the temperature of the baryons, $m_{p}$ the proton mass, $k_{B}$ the Boltzmann constant, and $k$ the wave vector of the Fourier density mode under consideration. For a matter-dominated universe with $\delta_{\mathrm{DM}} \propto a$, one finds

$$
\delta_{B}(t)=\frac{\delta_{\mathrm{DM}}(t)}{1+A k^{2}},
$$

where $A=3 / 2\left(k_{B} T_{0} / m_{p}\right)\left(t^{2} / a^{3}\right)$ with $T_{0}$ the temperature today (using matter dominance from decoupling to the present). Baryons are thus coupled tightly to dark matter on large scales, whereas the pressure support on small scales $\left(A k^{2} \ll 1\right)$ suppresses growth. If there was a primordial rotation of the dark matter of sufficient magnitude to cause vortex formation, then one would have to modify the driving term $\delta_{\mathrm{DM}}$ on the right-hand side of (6) to reflect the vortex structure. Given some characteristic vortex size (as in (5)) one would have to make the Fourier transform of a Swiss-cheese-like configuration of the dark matter. This would then give a new driving term and thus influence the structure formation in the baryons. There is a technical point here that we have ignored, which is that the Jeans analysis does not strictly apply to rotating systems. There would be corrections to this from Coriolis and centrifugal terms but we assume that these can (at least locally) be neglected. This assumption would of course have to be checked in more detailed investigations.

Since there is no evidence for primordial rotation, the discussion above is likely of little relevance to the universe we live in. The rotation that we observe in spirals must therefore originate in late-time events when the perturbations have entered the nonlinear regime and Jeans analysis is no longer useful. Estimates show that the galactic structures that we see today cannot not have formed at redshifts much larger than $z \sim 10$ [43]. Linear perturbation theory is expected to hold around decoupling which is $z \sim 1000$, so we are well beyond this approximation. The structure of baryons is also complicated by pressure and radiative terms, which means that hydrodynamical codes must be used. State of the art in structure formation is highly involved $\mathrm{N}$-body computer simulations that can calculate the evolution of the initial spectrum of perturbations into the nonlinear regime. However, these simulations do show that the gravitationally bound systems that arise tend to virialize on a fairly short time scale. In this paper we will not consider such advanced simulation techniques but merely consider what the effects of vortices could be on the virialized structures, since these are the ones that we observe to be rotating.

5.2. Influence on Virialization. Let us now address the question of the virialization process itself. The usual argument is that the bound system collapse under gravity with internal heating as a result. The virial theorem tells us that the internal kinetic energy should be half the gravitational potential. The systems therefore collapses until a radius is reached where this condition is fulfilled. However, when we introduce shortrange two-body repulsion for the BEC dark matter, the virial theorem is modified and becomes

$$
2 E_{\text {kin }}+E_{\text {grav }}+3 E_{\text {rep }}=0
$$

where $E_{\text {kin }}$ is the kinetic energy, $E_{\text {grav }}$ is the gravitational potential energy, and $E_{\text {rep }}$ is the energy arising from the twobody term. The latter contribution is easy to calculate for our zero-range potential and becomes

$$
E_{\text {rep }}=\frac{2 \pi \hbar^{2} a}{m} \int d \vec{r} n^{2},
$$

where $n(\vec{r})$ is the density of BEC dark matter. $N$-body simulations and observations tell us that objects virialize. So the contributions from $E_{\text {rep }}$ must be negligible in virialization to not jeopardize this fact. Consider the ratio

$$
\frac{E_{\text {rep }}}{E_{\text {grav }}}=-\frac{5}{2} \frac{a \hbar^{2}}{G R^{3} m^{3}} .
$$

Here we are assuming a uniform sphere for the matter distribution which gives $E_{\text {grav }}=-3 / 5 G M^{2} / R$. In order for $E_{\text {rep }}$ to not influence virialization, we need this ratio to be much smaller than 1 . However, since we have the relation between $a$ and $m$ in (3) this ratio becomes simply $-5 / 2 \pi^{2} \sim$ -0.25 , so we see that the repulsive interaction will be nonnegligible. We therefore have to reconsider how to apply the virial theorem. This we will do along the lines of the simple top-hat model as discussed in [43].

The virial theorem with inclusion of the repulsive twobody interaction and the use of (3) for $m$ yields

$$
\begin{aligned}
E_{\text {kin }} & =-\frac{1}{2}\left(+E_{\text {grav }}+3 E_{\text {rep }}\right)=-\left(\frac{1}{2}-\frac{15}{4 \pi^{2}}\right) E_{\text {grav }} \\
& \approx-0.12 E_{\text {grav }}
\end{aligned}
$$

We see that the internal kinetic energy is a considerably smaller fraction of the gravitational energy than for standard virial consideration with $a=0$. If we have a sphere of matter with radius $r_{\max }$ that starts to collapse from rest, then from conservation of energy one can easily estimate that the virial theorem will be satisfied when $r \approx r_{\max } / 1.14$, whereas for 
$a=0$ would be $r=r_{\max } / 2$ [43]. Virialization thus predicts a less compact system in the $a \neq 0$ case. This means that when gravitational objects become virialized they will have densities that are about $27 \rho_{b}$, as compared to the $a=0$ estimate of $150 \rho_{b}$ [43]. The two-body repulsive interactions are thus seen to yield less dense objects, something that could be very good in terms of getting less dense halos than some simulations produce and that are at odds with observations. To test whether this helps would require full simulations with ultralight BEC dark matter.

$\mathrm{N}$-body simulations predict that virialization occurs at densities above $150 \rho_{b}$ (estimates put the value close to $400 \rho_{b}$ [43]), which is an order of magnitude larger than the calculation above. Ultralight BEC dark matter would therefore seem to be ruled out. However, the above results are only as good as the assumptions we use in their derivation. In particular, the use of (3) is based on the Lane-Emden result of (2). We know that halos do not have this profile. We should therefore not put too much emphasis on the mass $m$ in (3), but rather leave $m$ as a parameter. If we assume that $E_{\mathrm{rep}}=-\kappa E_{\text {grav }}$, one can invert the virialization analysis of [43] to get an estimate of $\kappa$ if we are to get virialized densities of $\gtrsim 400 \rho_{b}$. This yields $\kappa \lesssim 0.1$. For this to be fulfilled, (10) tells us that $a / m_{22}^{3} \lesssim 4 \cdot 10^{-44} \mathrm{fm} / \mathrm{eV}^{3}$ with $a$ in Fermi and $m_{22}=m / 10^{-22} \mathrm{eV}$. This shows that we can actually accommodate that $\mathrm{N}$-body results on virialization are fulfilled by having a small value of $a$ for ultralight Bose particles. Such small values for $a$ are also consistent with the values predicted from the arguments in earlier sections of $a \sim 10^{-61} \mathrm{fm}$.

5.3. Influence on Galactic Rotation Curves. Another interesting issue is whether a dark matter vortex lattice can influence the galactic rotation curve. These curves are key pieces of evidence for the existence of dark matter due to their flat (or slightly increasing) behavior at large radii where standard Newtonian theory including only luminous matter predicts a sharp decrease with radius. The galactic rotation velocity is calculated from the basic formula [42]

$$
v^{2}(r)=\frac{G M(r)}{r}=\frac{4 \pi G}{r} \int_{0}^{r} d x x^{2} \rho(x),
$$

where $\rho(r)$ is the total mass contribution from dark and luminous matter. In the present work we assume that the dark halo contribution has the form

$$
\rho_{h}(r)=\frac{\rho_{0}}{1+\left(r / r_{0}\right)^{\gamma}},
$$

where $r_{0}$ is related to the core radius and $\gamma$ is the exponent determining the large distance behavior. This form avoids the small distance cusp that BEC DM presumably solves [12-15]. The rotation curve can now be calculated from the simple formula $v^{2}=v_{\text {dark }}^{2}+v_{\text {lum }}^{2}$, where $v_{\text {lum }}$ is the contribution from the luminous matter in the bulge, disk, and surrounding gas.

Experiments with rotating atomic BEC produce triangular vortex lattices. Theoretically, this is is the minimum energy configuration, with the square lattice slightly higher in energy [44]. Recent studies including long-range dipoledipole interactions between the bosons find that other configurations are preferred as the long-range forces increase [45], and we speculate that similar results can arise for the $1 / r$ gravitational force. However, since it is unclear which kind of lattice would arise in the galactic halos, we have explored both triangular and square lattices. We also mention that square lattices are commonly seen in twocomponent condensates [46], which would be relevant for multiple species BEC DM.

The vortex lattice is modeled as an array of zero-density tubular regions of square cross section in the DM mass density. We have thus ignored the spherical shape and the smooth fall-off to zero density that the more realistic vortex cores would have. We have checked that this simplification did not have significant effects on our results. Due to this implementation, the vortex size represents an effective coherence length, simply related to $\xi$. Keep in mind that the square shape can cause some rather sharp features in the calculated rotation curves which are smoothed for more realistic profiles. To find the rotation velocity we have numerically integrated this "Swiss-cheese" halo along with the luminous contributions. In effect the method used assumes that the lattice rotates with the system, which is what is seen in cold gas experiments as well. To demonstrate the effect of the vortex lattice, we show in Figure 1 a number of rotation curves based solely on the DM. These have been calculated for vortex size $5 \mathrm{kpc}$ and vortex-vortex distances of 1 and $2.5 \mathrm{kpc}$. These parameters are optimal in order to get the oscillations at short distances shown in Figure 1. For smaller core sizes the effect vanishes and for larger ones the scale of wiggles is too large to fit observations.

At this point we mention that we have used a cutoff on the vortex lattice for small radii (of order the halo size $r_{0}$ ), effectively ignoring vortices that are located in the halo center. With this cutoff we can fit the observation of large constant density in the center of the galaxies. This appears reasonable since luminous matter should be dominant in the innermost region. Another issue is the density of vortices. In our simple model we have assumed this to be constant with the use of regular lattices but there could be variations [47].

The wiggles found in the velocity curves with vortices present are quite interesting in relation to observations. Here one also sees distinct wiggling features in the plots $[11,17]$. In [11] the data from NGC2998 was explained by postulating the BEC dark matter to be in an excited condensate state which has several zeros in the wave function. When this is translated into a dark matter distribution it gives areas of low density in the halo. In the velocity curves this will of course have similar effects as the vortex lattice with its empty cores. However, with vortices one can get wiggles without having to explain how the excitation occurred and why the condensate should be in a particular excited state at present. One would of course also have to include the luminous matter for a real comparison to data, and for small radii this will presumably dominate the rotation curves [48]. Below we will include the effects of the luminous component explicitly when comparing to observations.

Before embarking on a comparison to observations, we note a number of problems with this model. First, we have assumed that the vortex cores have the same shape at all 


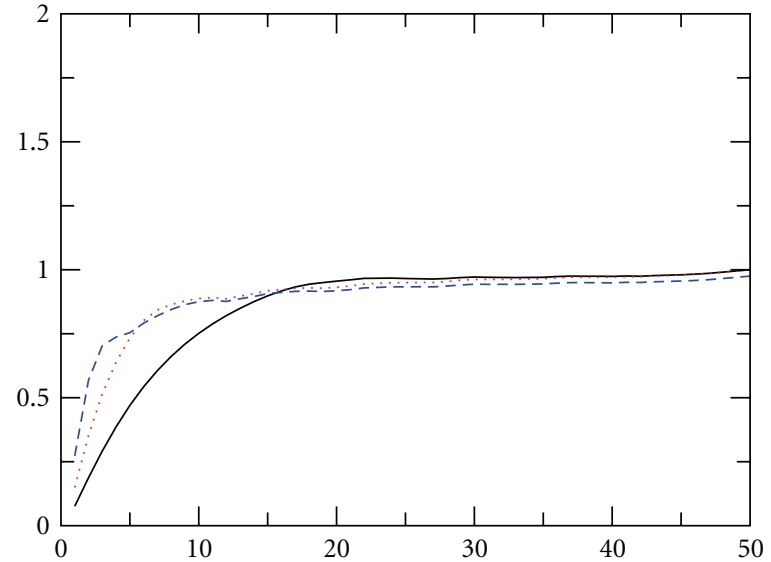

(a)

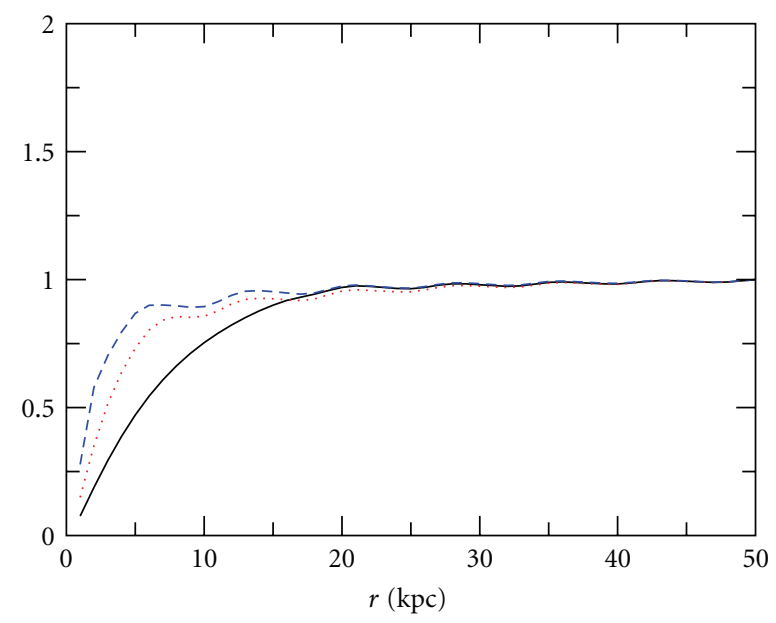

(c)

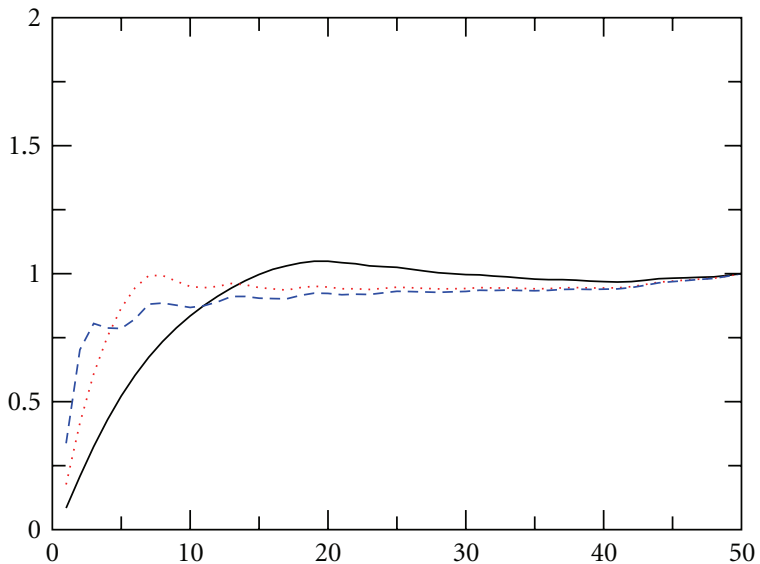

(b)

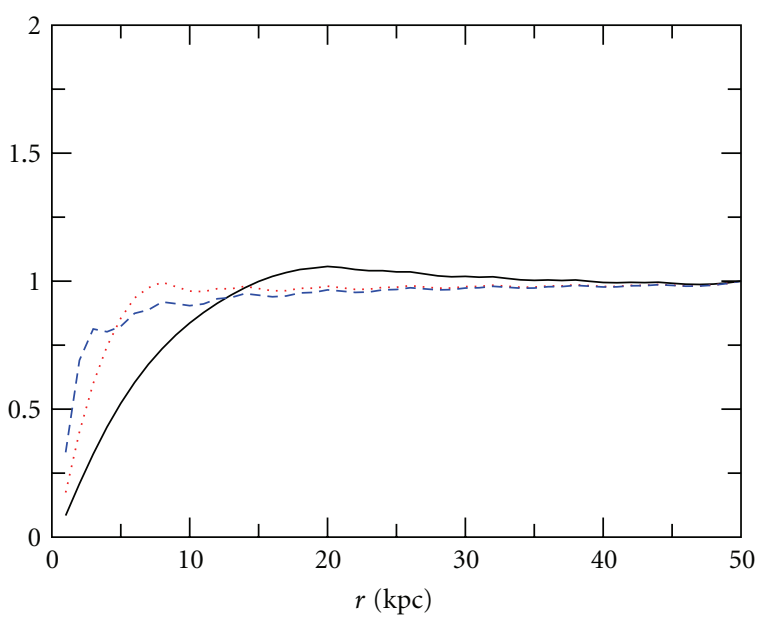

(d)

FIGURE 1: Rotation curves based on BEC DM with a vortex lattice for different halo sizes $r_{0}=8$ (full), $r_{0}=4$ (dotted), and $r_{0}=2 \mathrm{kpc}$ (dashed). The vortex core size is $5 \mathrm{kpc}$. In the left column the distance between vortices is $2.5 \mathrm{kpc}$, whereas it is $1 \mathrm{kpc}$ in the right column. Upper row is for a triangular lattice, and the lower one is for a square lattice.

radii, independent of the local halo distribution. This is likely unrealistic as we would expect some effects of the finite size toward the edge. Secondly, for the coherence length to approach 5-10 kpc, from (5), we would need either $a \sim$ $10^{-65} \mathrm{fm}$ or an increase in $m_{22}$ by four orders of magnitude (or a combination of both). We estimated earlier that existing suggestions for the parameters of ultralight BEC dark matter of $a \sim 10^{-61} \mathrm{fm}$ and $m \sim 10^{-22} \mathrm{eV}$ gives $\xi \sim 0.05 \mathrm{kpc}$, which would be impossible to see in velocity curves. So it would seem that we need some fine-tuning of $m$ and $a$ to get any effect. This we will be addressed below.

5.4. Comparison to Spiral Galaxies. An interesting feature of the BEC DM model is the oscillations in the rotation velocity at small radii, and we now compare the model to spirals that show pronounced oscillatory features. Both dark and luminous matter play important roles in modeling spirals, and we therefore include the luminous component based on recommended values from various observation as described below. Note that we allow the dark matter component and the details of the vortices to vary in order to best describe observations. For simplicity, we ignore the feedback effect on the luminous matter of changing the dark component. Since luminous matter dominates the rotation curve at small radii and dark matter at larger radii in our model, this should be a fair approximation.

We consider a sample of spirals that have been used in BEC dark matter studies previously $[11,16,17]$. In Figure 2 we show data for the Sc spiral NGC2998 [49]. The bulge was assumed to give a constant contribution for $r<3 \mathrm{kpc}$ and then fall as $r^{-1 / 2}$ [50], whereas the disk and gas are combined into the functional form $(r /(r+b))^{6}$ of range $b$ [17]. The magnitudes of these nondark contributions were scaled to reproduce those in [50], whereas the DM contribution is varied to best describe the observations. It can be seen in Figure 2 that a square lattice is closer to the data. In particular, we see good agreement between maxima and minima of the curves, although the magnitudes are not well reproduced, particularly at larger radii. We find that the oscillations in the model are directly related to the vortex 


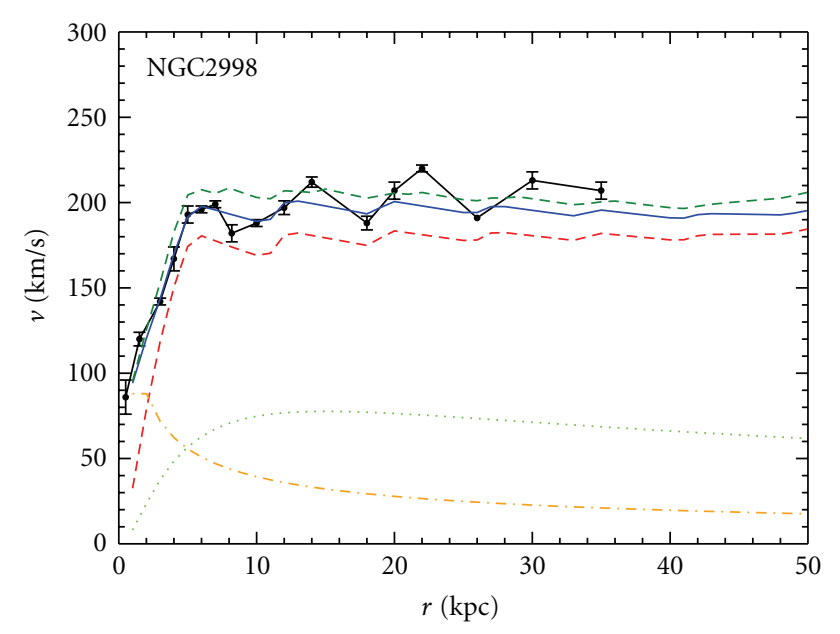

FIGURE 2: Rotation curve data for the spiral galaxy NGC2998 (dots with uncertainties) and calculation with a square (thick line) and triangular (short dashed) BEC vortex lattice in the DM component. Shown are also the individual contributions from the bulge (dotdashed), disk + gas (dotted), and DM for the square lattice case (long dashed). The parameters used are $r_{0}=5.0, \gamma=2.15, b=3.0$, $L_{v}=6.5$, and $d=1.0$ (all lengths in $\mathrm{kpc}$ ).

core size, $L_{v}$, and lattice distance, $d$, and the characteristic length of the wiggles observed in the rotation curves can thus be matched in the present model by careful choice of parameters. However, as explained before the main source of the oscillatory behavior is considered to be baryonic due to the presence of spiral arms.

To further explore the model, we consider observations from two Sc spirals, NGC753 and NGC801 [49], a dwarf spiral, NGC1560 [51], and a thin disk spiral, NGC3198 [52] in Figure 3. The nondark components are modeled as for NGC2998 above. Notice that NGC1560 and NGC3198 have no bulge. The DM for NGC753 and NGC801 assumes a square vortex lattice (a triangular lattice compares worse with data), and we see that this gives a better agreement with the observed wiggles than for NGC2998, but still with discrepancies between minima and maxima. This could likely be resolved, if one makes the vortex density nonuniform as suggested in [47]. In the dwarf case of NGC1560, we see that our model can reproduce the observed kink in the curve, in this case even better with the triangular lattice. This feature was not reproduced in previous fits to BEC DM [16, 17]. In the thin disk spiral NGC3198 our model only gives a small improvement over previous studies, and square and triangular results are practically the same. There are some discrepancies at large radii, which would likely be reconciled by a better description of the gas component.

The BEC DM model can accomodate data on different spiral types, and the parameters used are very similar. The vortex cores are $5.0-6.5 \mathrm{kpc}$ in size with a lattice spacing of $1-2 \mathrm{kpc}$. We note that experiments have shown that the core and vortex-vortex distance can be of similar magnitude [53] in trapped condensates, although the core size is smaller than the distance. The theoretical vortex density expected in uniform condensates is given by $n_{v}=m \Omega / \pi \hbar=0.05\left(m / 10^{-22} \mathrm{eV}\right)\left(\Omega / 10^{-16} \mathrm{~s}^{-1}\right) \mathrm{kpc}^{-2}$. For the spirals considered here we get $n_{v} \sim 0.10-0.13 \mathrm{kpc}^{-2}$. The total number of vortices is then $N=\pi R^{2} n_{v} \sim 30-1000$ for radii $R \sim 10-50 \mathrm{kpc}$. For the square lattice this gives about 45 vortices within a distance of $50 \mathrm{kpc}$ using the size and distance quoted above, which is a reasonable halo size for the Sc spirals discussed. Our model parameters are thus not completely untenable. With smaller cores we could get larger densities or have larger distance between vortices. However, we have found that in order to obtain the wiggles in the rotation curves the core size has to be roughly equal to the length between maxima and minima in the observations. In NGC3198 we have used a larger distance of $2.0 \mathrm{kpc}$ to get a good fit, consistent with its rotational velocity being slightly lower than that of the others. Also for the dwarf NGC1560 we have used a smaller core size. This gives a larger vortex density which is consistent with a slightly larger rotational rate compared to the Sc samples.

The wiggles in the rotation curves are located at short radii and studies have shown them to be correlated with the spiral arms $[28,29]$, both facts strongly indicating baryonic processes as the origin. The current model implies that there could be additional effects in an ultralight BEC dark matter scenario. Assuming that the wiggles are caused by baryonic matter only, we can use the current model to extract bounds on the parameters from the lack of vortex lattice effects in observations. This would in turn influence other models of BEC DM through limits on boson masses and two-body coupling terms. In particular, the absence of large wiggles produced by vortex lattices would give a lower limit on the two-body repulsion of the bosons.

\section{Fine-Tuning Issues}

The parameters of importance to the success of an ultralight BEC dark matter model are naturally the mass, which must be extremely small, and, for the self-interacting scenario, the strength of the two-body repulsion between the condensed particles. In [19] BEC dark matter is suggested to arise from a single scaler field coupled to gravity undergoing spontaneous symmetry breaking to acquire a vacuum expectation value. This produces a cosmological constant $\Lambda$. The breaking of symmetry is done via a Ginzburg-Landau potential with quadratic and quartic terms. This gives mass and interaction terms to the scalar field. If we now make the additional assumption that the vacuum expectation value, $\phi_{0}$, arises from a mechanism that preserves parity (so that third-order terms can be ignored), the interaction term is

$$
\frac{\left(m c^{2}\right)^{2} \phi^{4}}{4(\hbar c)^{2} \phi_{0}^{2}}=g \frac{\phi^{4}}{4},
$$

where we have restored constants of $c$ and $\hbar$. This term is of course merely the standard interaction term in the Gross-Pitaevskii theory of interacting condensed bosons. We therefore see that the self-interacting scenario emerges from this procedure.

The above scenario actually contains some additional information about the mass and interaction strength of the 


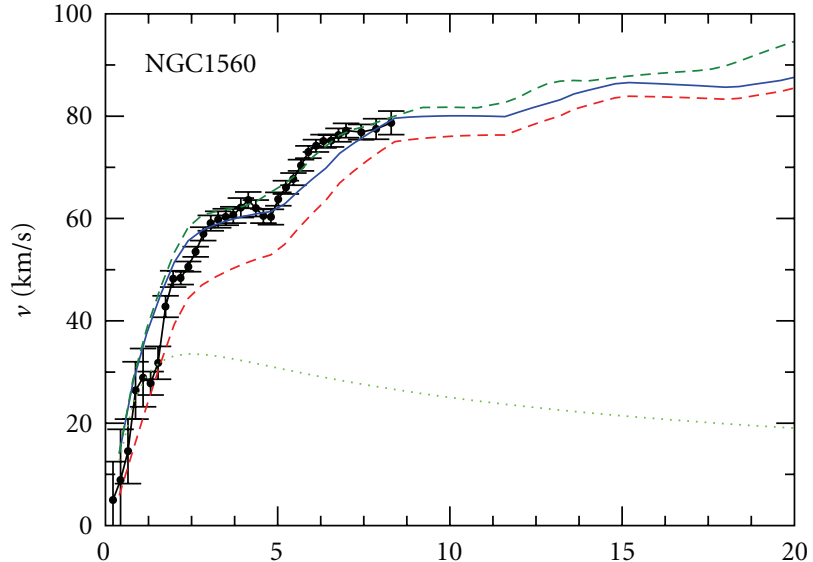

(a)

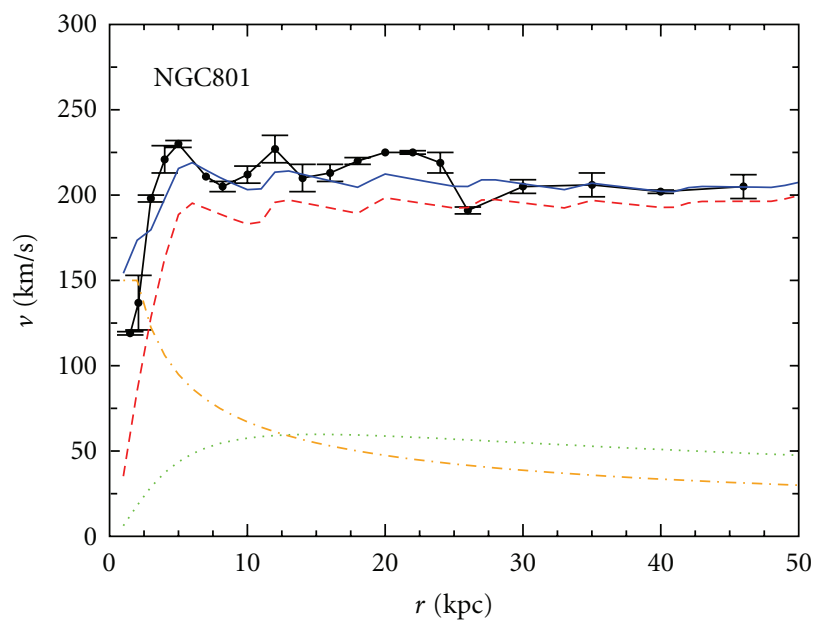

(c)

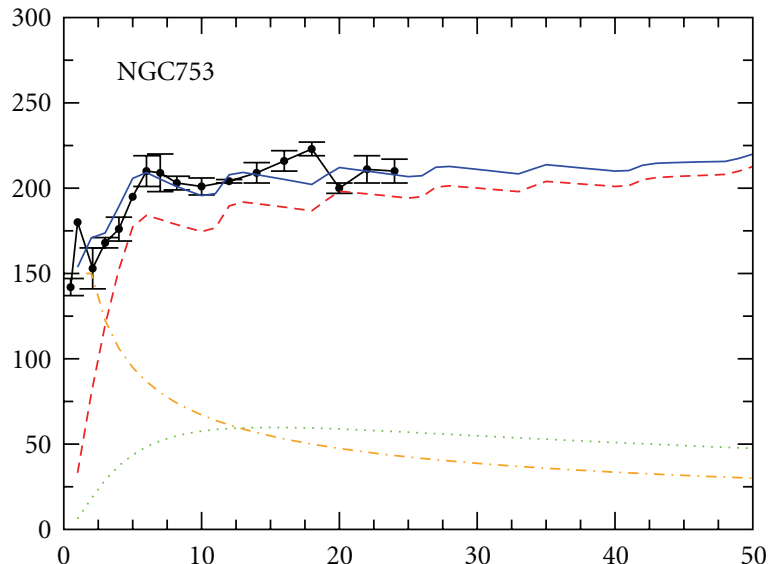

(b)

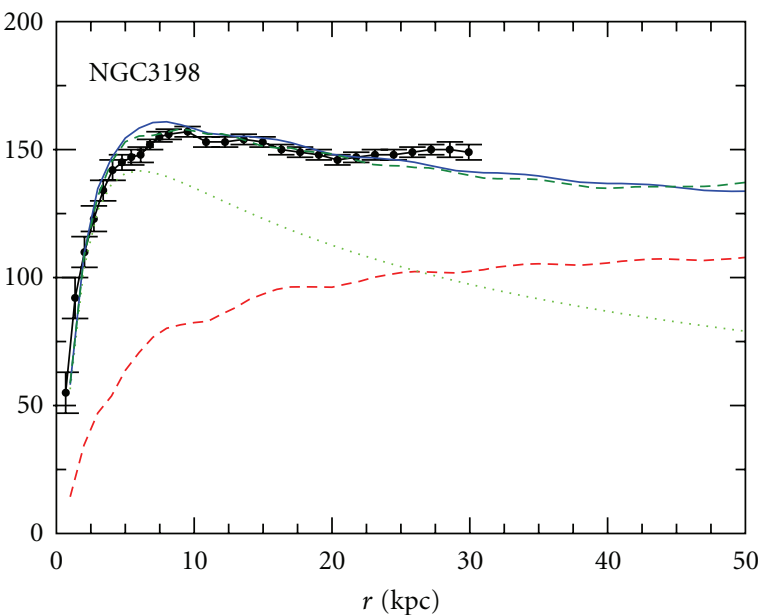

(d)

FIGURE 3: Rotation curves for NGC1560 (5.0, 2.15, 0.5, 5.0, 1.0), NGC753 (5.0, 2.00, 3.0, 6.5, 1.0), NGC801 (5.0, 2.15, 3.0, 6.5, 1.0), and NGC3198 (5.0, 2.00, 1.2, 6.5,2.0) compared to model calculations. The plot assignments are as in Figure 2. The parentheses give the parameters used in the format $\left(r_{0}, \gamma, b, L_{v}, d\right)$. Notice the change of radial distance scale for NGC1560.

scalar field. One can derive a relation between mass, the interaction strength, and the cosmological constant. As in the previous sections, we want to use $g=4 \pi \hbar^{2} a / m$ to express the interaction through the scattering length $a$. We have

$$
a=\frac{m^{5} G}{4 \pi \hbar^{6} \Lambda}=7 \cdot 10^{-30} m_{22}^{5} \frac{\Lambda_{0}}{\Lambda} \mathrm{fm},
$$

where $\Lambda_{0}=0.7 \rho_{\text {crit }} \sim 10^{-52} \mathrm{~m}^{-2}$ is the best estimate of the cosmological constant from WMAP [3]. As is clearly seen, the value of $a$ in this model is quite far from the considerations of the previous sections, where $a \sim 10^{-61} \mathrm{fm}$ was found to be a favorable value. A mass of order $m$ $10^{-28} \mathrm{eV}$ would be required to get this latter value of $a$ (this is still within the bounds on ultralight dark matter discussed in [54]).

These considerations imply that the dark matter vortex lattice proposed here is far from generic and requires finely tuned parameters to work. However, the model of spontaneous symmetry breaking discussed in [19] is also highly speculative. The cause of the observed dark energy is still unknown, and it is therefore reasonable to consider scenarios where the relation in (15) is not valid. This can easily be done by considering more general potentials than the standard Ginzburg-Landau scalar potential. The breaking of symmetry could then be accomplished in a manner that allows other relations between $m$ and $a$. This could allow other ranges of the parameters and perhaps make way for dark matter BEC vortex lattices without extreme finetuning. For lack of a better understanding, the cosmological constant, $\Lambda$, could be considered an outside parameter, and we want to address dark matter scenarios that are not necessarily from unified dark matter-dark energy models.

\section{Relevant Experiments and Numerical Simulations}

The present experimental situation in ultracold atomic gases also encourages the hope that one could possibly probe the consequences of bosonic dark matter and condensates 
in laboratory experiments. Many groups around the world can routinely produce condensates of bosons, degenerate Fermi gases, and also interesting mixtures of the two [55, 56]. Another feature is the extreme control experimentalists exercise over the interactions between the different atomic species. This is achieved through the use of Feshbach resonances that allows the tuning of the interactions strength over many orders of magnitude and also whether the atoms repel or attract each other.

A very interesting proposal is that one can tune the interatomic dipole-dipole interaction to resemble the gravitational force [27]. By illuminating the atomic clouds with a careful arrangement of multiple laser beams, it was suggested how to eliminate the $1 / r^{3}$ dependence of the dipole-dipole interaction and keep only the $1 / r$ part in the near zone. The force constant of the remaining term depends on the laser intensity and atomic polarizability. The resulting force is equivalent to the attraction between two opposite electric charges with $q \sim e / 2000$, so compared to electromagnetism it is not strong. However, in comparison, the tiny magnitude of gravity means that this force can be much larger than the normal gravitational force based on the atomic masses.

If this proposal is successfully implemented in experiment, one could therefore simulate gravitational forces between the gas particles of both Fermi and boson species, and with "effective" masses that are vastly different from the given atomic masses. This could very likely be a way to test the movement of particles during virialization and see how structures form. Comparison with observations and $N$-body simulations would then allow us to expand our knowledge of the evolution of structure in our universe. Since rotation is also routinely applied to the ultracold gases $[55,57]$, there would also be ways to test the scenario considered in this paper with the formation of vortex lattices. One could then imagine a mixture of clouds with bosonic particles as dark matter and then Fermions to represent luminous matter. This would allow the experimental exploration of the influence of BEC dark matter on structures in the luminous component. The ability to tune self-interactions and cross species interaction could also allow us to test effects of dark matter-normal matter interactions.

Although these experiments are extremely hard, the advances of recent years leave us hope that one could reach experimental capabilities that can explore some of these systems in the not too distant future. In fact, within traditional condensed matter the ideas of simulations for instance cosmological phenomena using experiments have been around for some [58], and this type of thinking naturally extends to ultracold atomic gases which are often seen as a simulator for condensed matter systems.

Alternatively, the dynamics can be simulation on a computer. Comparison of theory and experiment on ultracold atomic gases has shown that the Bose-Einstein condensates can be well described by the nonlinear Gross-Pitaevskii equation [18] which can be solved numerically with great precision (see [59] for details on state-of-the-art numerical techniques and references to relevant work). The inclusion of attractive $1 / r$ gravitational type potentials has been considered recently [60] in both a variational approach and in numerical simulations. Rotation should be possible to include in similar fashion. We speculate that to separate dark and luminous matter one can consider a mixture of two bosonic atoms that are rotated differently so as to allow for vortices in one but not the other component or simply a bose-Fermi mixture as also mentioned above. Issues of a potential thermal noncondensed part of the dark matter BEC could also be addressed numerically.

A recent example of the success of the Gross-Pitaevskii approach is worth mentioning. The atom ${ }^{52} \mathrm{Cr}$ has a large magnetic dipole moment that can be aligned by externally applied fields. This means that an attractive dipole-dipole force can be created that was predicted to produce collapse of a ${ }^{52} \mathrm{Cr}$ condensate. This has been experimentally observed and found to be in agreement with predictions from the Gross-Pitaevskii equation including the long-range force [61]. This implies that long-range forces can be accommodated by the Gross-Pitaevskii theory as well.

\section{Conclusions}

We have considered some models of dark matter where the main component is a bosonic particle with a very small mass of order $m \sim 10^{-22} \mathrm{eV}$. Naively, this gives a de Broglie wavelength that is of galactic proportion such that one can imagine the entire galactic dark matter halo being in the condensate ground state. We considered previous proposal with such particles with and without repulsive selfinteractions and found general consistency between these for certain ranges of the mass and interaction strength. Moreover, we considered the suggestion that superfluid BEC dark matter in rotation would likely also lead to vortices as seen in atomic BEC experiments.

In case of a repulsive self-interaction we argued that the vortex size should be determined locally by the coherence length of this repulsive interaction. This means that we have two scales in the problem: a galactic one, given by the de Broglie wavelength from the tiny mass, and a subgalactic one that is determined by the mass and the two-body interaction strength (characterized by the scattering length a). We explored the consequences of self-interactions on the virialization of gravitationally bound structures and found almost no effect for reasonable values of $m$ and $a$.

Under the assumption of dark matter being an ultralight BEC, the rotation of spiral galaxies would cause vortex lattices to form. We briefly addressed possible effects on the growth of perturbations in the linear regime, although this is probably not relevant since the rotation rate in the early universe is very small. We then considered possible effects of subgalactic vortices in the dark matter on the rotation velocity curves of virialized galaxies with standard dark matter halo profiles. Here we found that one can actually get substructure in the rotation curves that resemble some observations, but that this requires large vortex core size and small vortex-vortex distances. The mass and interaction strength needed to realize this were found to be fine tuned, but could possibly be accommodated in more general setups.

The present investigations and simple numerical experiments point to an interesting effect from bosonic dark 
matter. However, to fully explore the influence of vortex lattice formation and the feedback on structure formation in luminous matter one would need to consider an ultralight BEC dark matter component in large $N$-body simulations.

\section{Acknowledgments}

Discussions with H. O. U. Fynbo and S. Hannestad are highly appreciated. Thanks are also due to J. P. U. Fynbo for reading the draft and suggesting valuable improvements.

\section{References}

[1] A. G. Riess, A. V. Filippenko, P. Challis et al., "Observational evidence from supernovae for an accelerating universe and a cosmological constant," The Astronomical Journal, vol. 116, no. 3, pp. 1009-1038, 1998.

[2] S. Perlmutter, G. Aldering, G. Goldhaber et al., "Measurements of $\Omega$ and $\Lambda$ from 42 high-redshift supernovae," The Astrophysical Journal, vol. 517, no. 2, pp. 565-586, 1999.

[3] D. N. Spergel, L. Verde, H. V. Peiris et al., "First-year Wilkinson Microwave Anisotropy Probe (WMAP) observations: determination of cosmological parameters," The Astrophysical Journal, Supplement Series, vol. 148, no. 1, pp. 175-194, 2003.

[4] V. V. Zhytnikov and J. M. Nester, "Can galactic observations be explained by a relativistic gravity theory?" Physical Review Letters, vol. 73, no. 22, pp. 2950-2953, 1994.

[5] L. Bergström, "Non-baryonic dark matter: observational evidence and detection methods," Reports on Progress in Physics, vol. 63, no. 5, pp. 793-841, 2000.

[6] J. M. Overduin and P. S. Wesson, "Dark matter and background light," Physics Reports, vol. 402, no. 5-6, pp. 267-406, 2004.

[7] N. J. C. Spooner, "Direct dark matter searches," Journal of the Physical Society of Japan, vol. 76, no. 11, Article ID 111016, 2007.

[8] G. Dvali, G. Gabadadze, and M. Shifman, "Ultralight scalars and spiral galaxies," Modern Physics Letters A, vol. 16, no. 8, pp. 513-530, 2001.

[9] R. Kallosh, "Supergravity, M theory and cosmology," arXiv:hep-th/0205315v1.

[10] C. T. Hill, D. N. Schramm, and J. N. Fry, "Cosmological structure formation from soft topological defects," Comments on Nuclear and Particle Physics, vol. 19, p. 25, 1989.

[11] S. J. Sin, "Late-time phase transition and the galactic halo as a Bose liquid," Physical Review D, vol. 50, no. 6, pp. 3650-3654, 1994.

[12] W. Hu, R. Barkana, and A. Gruzinov, "Fuzzy cold dark matter: the wave properties of ultralight particles," Physical Review Letters, vol. 85, no. 6, pp. 1158-1161, 2000.

[13] J. Goodman, "Repulsive dark matter," New Astronomy, vol. 5, no. 2, pp. 103-107, 2000.

[14] P. J. E. Peebles, "Fluid dark matter," The Astrophysical Journal, vol. 534, no. 2, pp. L127-L129, 2000.

[15] F. E. Schunck and E. W. Mielke, "General relativistic boson stars," Classical and Quantum Gravity, vol. 20, no. 20, pp. R301-R356, 2003.

[16] F. E. Schunck, "A scalar field matter model for dark halos of galaxies and gravitational redshift," arXiv:astro-ph/ $9802258 \mathrm{v} 1$.

[17] C. G. Böhmer and T. Harko, "Can dark matter be a BoseEinstein condensate?" Journal of Cosmology and Astroparticle Physics, vol. 2007, no. 6, article 025, 2007.
[18] L. P. Pitaevskii and S. Stringari, Bose-Einstein Condensation, Oxford University Press, New York, NY, USA, 2003.

[19] M. P. Silverman and R. L. Mallett, "Dark matter as a cosmic Bose-Einstein condensate and possible superfluid," General Relativity and Gravitation, vol. 34, no. 5, pp. 633-649, 2002.

[20] J. Mielczarek, T. Stachowiak, and M. Szydłowski, "Vortex in axion condensate as a dark matter halo," International Journal of Modern Physics D, vol. 19, no. 11, pp. 1843-1855, 2010.

[21] G. Chapline and P. O. Mazur, "Superfluid picture for rotating space-times," arXiv:gr-qc/0407033v2.

[22] G. Chapline, E. Hohlfeld, R. B. Laughlin, and D. I. Santiago, "Quantum phase transitions and the breakdown of classical general relativity," International Journal of Modern Physics A, vol. 18, no. 21, pp. 3587-3590, 2003.

[23] E. J. Yarmchuk, M. J. V. Gordon, and R. E. Packard, "Observation of stationary vortex arrays in rotating superfluid helium,” Physical Review Letters, vol. 43, no. 3, pp. 214-217, 1979.

[24] K. W. Madison, F. Chevy, W. Wohlleben, and J. Dalibard, "Vortex formation in a stirred Bose-Einstein condensate," Physical Review Letters, vol. 84, no. 5, pp. 806-809, 2000.

[25] J. R. Abo-Shaeer, C. Raman, J. M. Vogels, and W. Ketterle, "Observation of vortex lattices in Bose-Einstein condensates," Science, vol. 292, no. 5516, pp. 476-479, 2001.

[26] P. Engels, I. Coddington, V. Schweikhard, and E. A. Cornell, "Vortex lattice dynamics in a dillute gas BEC," Journal of Low Temperature Physics, vol. 134, no. 1-2, pp. 683-688, 2004.

[27] D. O'Dell, S. Giovanazzi, G. Kurizki, and V. M. Akulin, "Bose-Einstein condensates with $1 / \mathrm{r}$ interatomic attraction: electromagnetically induced 'gravity', Physical Review Letters, vol. 84 , no. 25 , pp. 5687-5690, 2000.

[28] C. Beauvais and G. Bothun, "Precision velocity fields in spiral galaxies. I. Noncircular motions and RMS noise in disks," The Astrophysical Journal, Supplement Series, vol. 125, no. 1, pp. 99-121, 1999.

[29] I. Dicaire, C. Carignan, P. Amram et al., "H $\alpha$ kinematics of the spitzer infrared nearby galaxies survey-II," Monthly Notices of the Royal Astronomical Society, vol. 385, no. 2, pp. 553-605, 2008.

[30] T. Matos and L. A. Ureña-López, "Further analysis of a cosmological model with quintessence and scalar dark matter," Physical Review D, vol. 63, no. 6, Article ID 063506, 2001.

[31] G. Baym, J.-P. Blaizot, M. Holzmann, F. Laloë, and D. Vautherin, "The transition temperature of the dilute interacting Bose gas," Physical Review Letters, vol. 83, no. 9, pp. 17031706, 1999.

[32] J. D. Barrow, R. Juszkiewicz, and D. H. Sonoda, "Universal rotation: how large can it be?" Monthly Notices of the Royal Astronomical Society, vol. 213, p. 917, 1985.

[33] A. Arbey, J. Lesgourgues, and P. Salati, "Galactic halos of fluid dark matter," Physical Review D, vol. 68, no. 2, Article ID 023511, 2003.

[34] K. Lanczos, "Über eine stationäre Kosmologie im Sinne der Einsteinschen Gravitationstheorie," Zeitschrift für Physik, vol. 21, no. 1, pp. 73-110, 1924.

[35] G. Gamow, “Rotating universe?” Nature, vol. 158, no. 4016, p. $549,1946$.

[36] K. Gödel, "An example of a new type of cosmological solutions of einstein's field equations of gravitation," Reviews of Modern Physics, vol. 21, no. 3, pp. 447-450, 1949.

[37] S. Hawking, "On the rotation of the universe," Monthly Notices of the Royal Astronomical Society, vol. 142, p. 129, 1969.

[38] J. Silk, "The instability of a rotating universe," Monthly Notices of the Royal Astronomical Society, vol. 147, pp. 13-19, 1970. 
[39] P. J. E. Peebles, "Origin of the angular momentum of galaxies," The Astrophysical Journal, vol. 155, p. 393, 1969.

[40] A. G. Doroshkevich, "Spatial structure of perturbations and origin of galactic rotation in fluctuation theory," Astrofizika, vol. 6, p. 581, 1970.

[41] S. D. M. White, "Angular momentum growth in protogalaxies," The Astrophysical Journal, vol. 286, pp. 38-41, 1984.

[42] T. Padmanabhan, Theoretical Astrophysics, vol. III, Cambridge University Press, 2002.

[43] M. S. Longair, Galaxy Formation, Springer, Berlin, Germany, 1998.

[44] V. K. Tkachenko, "On vortex lattices," Soviet Physics-JETP, vol. 22, p. 1282, 1966.

[45] N. R. Cooper, E. H. Rezayi, and S. H. Simon, "Vortex lattices in rotating atomic bose gases with dipolar interactions," Physical Review Letters, vol. 95, no. 20, Article ID 200402, 2005.

[46] V. Schweikhard, I. Coddington, P. Engels, S. Tung, and E. A. Cornell, "Vortex-lattice dynamics in rotating spinor boseeinstein condensates," Physical Review Letters, vol. 93, no. 21, Article ID 210403, 2004.

[47] R. P. Yu and M. J. Morgan, "Vortices in a rotating dark matter condensate," Classical and Quantum Gravity, vol. 19, no. 17, pp. L157-L166, 2002.

[48] P. Salucci, "The mass distribution in Spiral galaxies," Proceedings of the International Astronomical Union Symposium, vol. 3, no. 244, pp. 53-62, 2007.

[49] V. C. Rubin, D. Burstein, W. K. Ford Jr., and N. Thonnard, "Rotation velocities of 16 SA galaxies and a comparison of Sa, Sb, and SC rotation properties," The Astrophysical Journal, vol. 289, pp. 81-98, 1985.

[50] S. M. Kent, "Dark matter in spiral galaxies. I-galaxies with optical rotation curves," The Astronomical Journal, vol. 91, pp. 1301-1327, 1986.

[51] A. H. Broeils, "The mass distribution of the dwarf spiral NGC 1560," Astronomy \& Astrophysics, vol. 256, no. 1, pp. 19-32, 1992.

[52] K. G. Begeman, "H I rotation curves of spiral galaxies. I-NGC 3198," Astronomy \& Astrophysics, vol. 223, pp. 47-60, 1989.

[53] J. R. Abo-Shaeer, C. Raman, J. M. Vogels, and W. Ketterle, "Observation of vortex lattices in Bose-Einstein condensates," Science, vol. 292, no. 5516, pp. 476-479, 2001.

[54] S. M. Carroll, "Quintessence and the rest of the world: suppressing long-range interactions," Physical Review Letters, vol. 81, no. 15, pp. 3067-3070, 1998.

[55] I. Bloch, J. Dalibard, and W. Zwerger, "Many-body physics with ultracold gases," Reviews of Modern Physics, vol. 80, no. 3, pp. 885-964, 2008.

[56] S. Giorgini, L. P. Pitaevskii, and S. Stringari, "Theory of ultracold atomic Fermi gases," Reviews of Modern Physics, vol. 80, no. 4, pp. 1215-1274, 2008.

[57] A. L. Fetter, "Rotating trapped Bose-Einstein condensates," Reviews of Modern Physics, vol. 81, no. 2, pp. 647-691, 2009.

[58] W. H. Zurek, "Cosmological experiments in condensed matter systems," Physics Report, vol. 276, no. 4, pp. 177-221, 1996.

[59] P. Muruganandam and S. K. Adhikari, "Fortran programs for the time-dependent Gross-Pitaevskii equation in a fully anisotropic trap," Computer Physics Communications, vol. 180, no. 10, pp. 1888-1912, 2009.

[60] H. Cartarius, T. Fabčič, J. Main, and G. Wunner, "Dynamics and stability of Bose-Einstein condensates with attractive $1 / \mathrm{r}$ interaction," Physical Review A, vol. 78, no. 1, Article ID 013615, 2008.

[61] T. Lahaye, J. Metz, B. Fröhlich et al., "d-wave collapse and explosion of a dipolar bose-einstein condensate," Physical Review Letters, vol. 101, no. 8, Article ID 080401, 2008. 

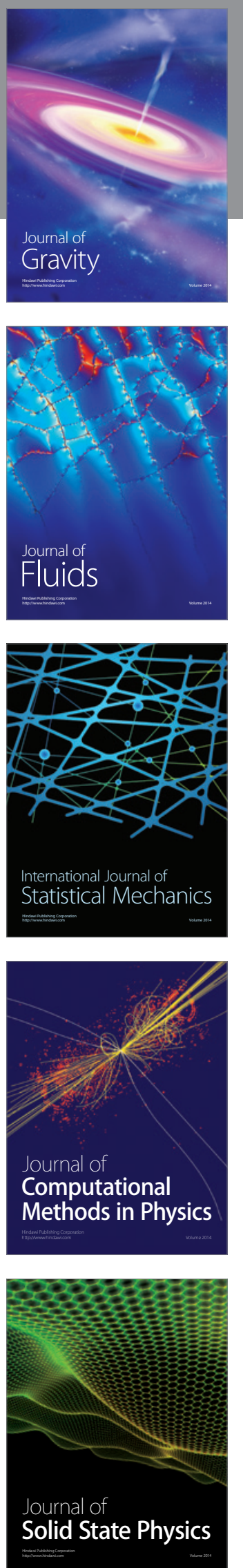

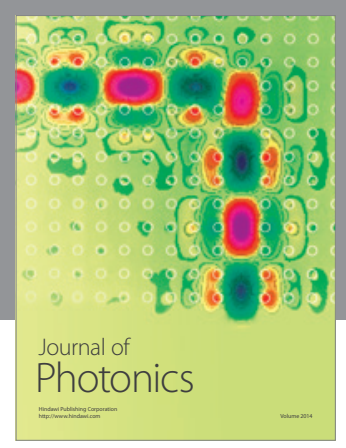

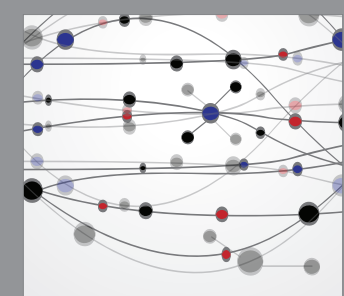

The Scientific World Journal
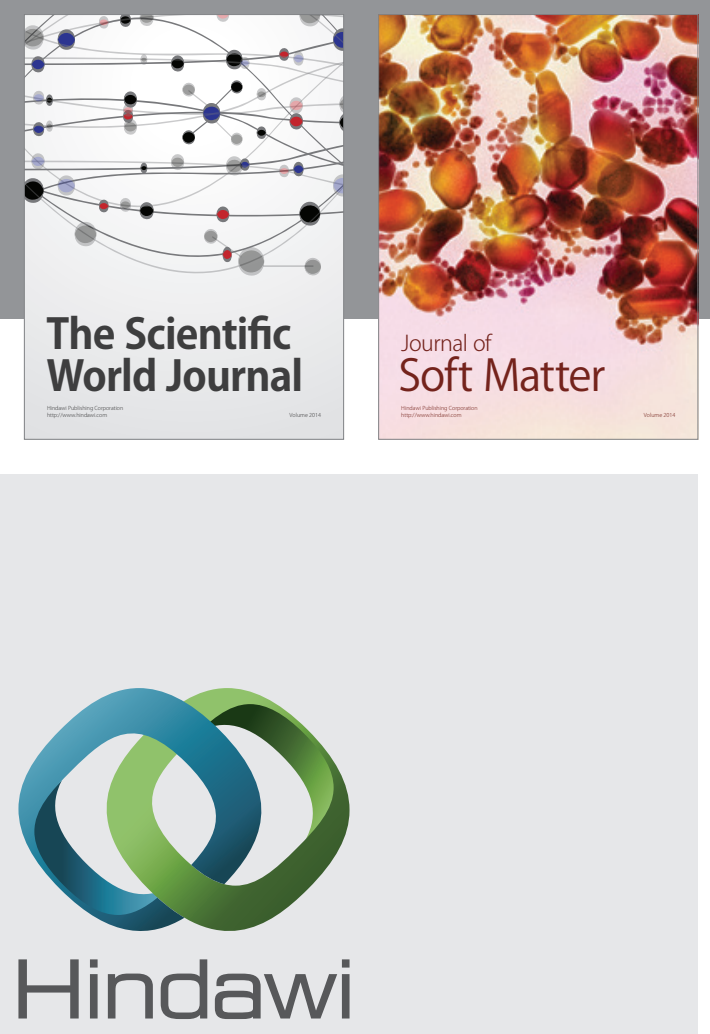

Submit your manuscripts at

http://www.hindawi.com
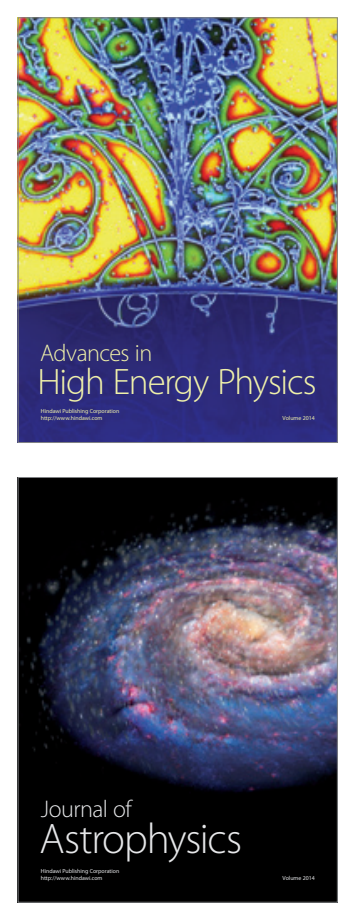
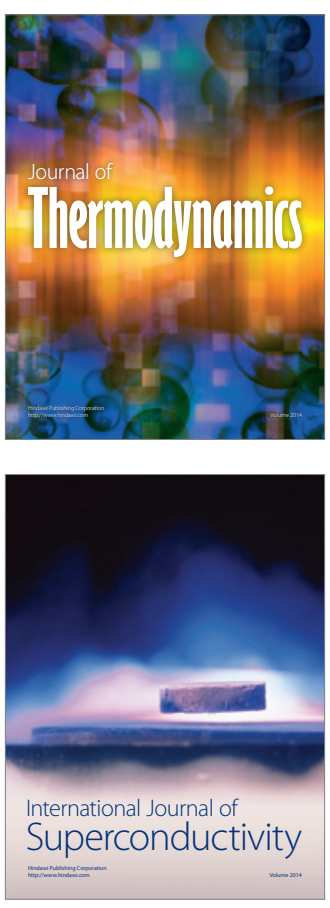
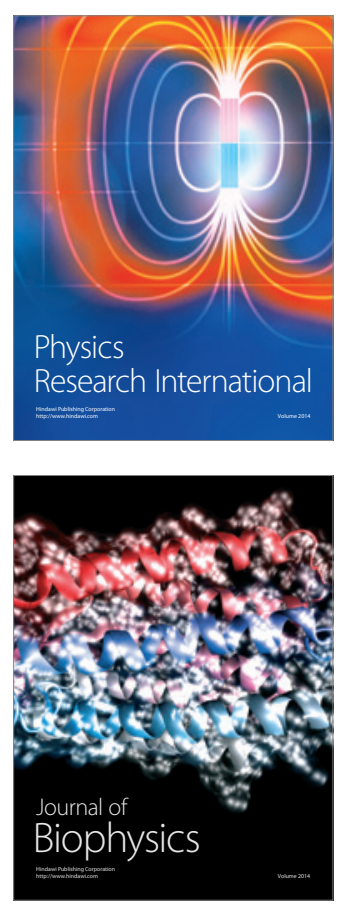
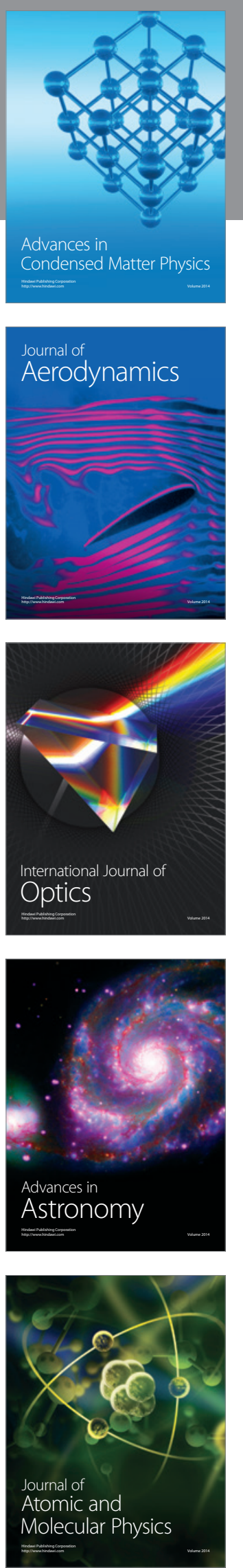